\title{
35. Unusual Cases of Chronic Subdural Hematoma
}

\author{
Jun-ichi KaWAFUCHI \\ Neurosurgical Department, Gunma University School of Medicine
}

The author has discussed the mechanism on production of chronic sub dural hematoma previously. Our conception was as follows: After a mild hear injury, blood oozes out into the subdural space and then reactive granulation tissur arises innerside of the dura mater which gradually over the clot and finally envelop: it. It is with further confirmation of the conception that this report deals.

Case 1 and 2 were 48 and 34 year old man, who had both subdural anc intracerebral hematomas after head injuries. The subdural hematomas were completely organized. In case 1 surface brain damageg was confirmed at the cranitomy.

Case 3. A 49-year-old man was admitted one month after a episode of spontaneous subarachnoid hemorrhage. A saccular aneurysm in the right middle cerebral artery and a semilunar radiolucency characteristic of chronic subdural hematoma over the right hemisphere were revealed angiographically. The aneurysm was coated with EDH adhesive and the completely organized hematoma membrane was removed.

Case 4. A 6 year old boy; About one year ago a large craniopharyngioma was evacuated with right transfrontal craniotomy. Two months prior to second admission he complained of headache and visual disturbance. Reoperation was carried out and the tumor was almost totally removed. At this exploration a $0.5 \mathrm{~mm}$ thick completely organized whitish subdural membrane containing no blood component was found over the previous explored frontal region.

\section{Traumatic Intracranial Space Occupying Lesions without Increased Intracranial Pressure or Focal Signs}

\author{
Minoru Tsunoda, Tamotsu Takeda and Toshiak Machida \\ Department of Neurosurgery, Sapporo City Hospital
}

In the out-patient clinic we have many chances to examine patients, complaining of headache etc., who have already passed the acute stage of head injuries.

During the past one and half years we experienced 10 cases (20\% of 49 cases of the traumatic intracranial space occupying lesion) who developed intracranial space occuying lesions without increased intracranial pressure or focal signs.

The final diagnosis was made by the carotid angiography and the operation. 